\title{
A Reasoning System about Bi-Agent Knowledge Sharing
}

\author{
Jinsheng Gao ${ }^{1, a}$,Changle Zhou ${ }^{2,3, b}$ \\ ${ }^{1}$ Department of philosophy, Xiamen University, Xiamen, Fujian, China, 361005. \\ ${ }^{2}$ Fujian Keylab of The Brain-like Intelligent Systems, Xiamen University, China,361005. \\ ${ }^{3}$ Department of CognitiveScience and Technology, Xiamen University, Fujian, China, 361005. \\ aemail: hi.Jackson@163.com, bemail:dozero@xmu.edu.cn,
}

Keywords: Dynamic epistemic logic; Common knowledge; Knowledge sharing

\begin{abstract}
In the field of artificial intelligence and computer science, Dynamic Epistemic Logic (DEL) is often used to model the knowledge, the inference in multi-agentknowledge, the action reasoningin game, and so on. This is because, unlike the general static epistemic logic, the dynamic perception can express the knowledge update in the multi-agent interaction. This paper,after analyzing some kinds of knowledge involved in the multi-agent interaction, proposes a Knowledge Sharing System (KSS) that extends the epistemic language with a new operator of 'knowledge sharing' for bi-agent interaction. Its soundness and completeness will beproved, and some properties about it will be analyzed in this paper.This system offers a clear presentation of how an agent gets to understand the other's intentions through the action of "sharing", which allows further interaction.
\end{abstract}

\section{Introduction}

Knowledge is an ancient and complex concept, but also an actively, frequently studied in many disciplines. Every major subject of artificial intelligence there are perception, planning, reasoning, learning and communication can be interpreted as the concept of knowledge, therefore knowledge lies at the head in artificial intelligence [1]. The definition of knowledge involves a wide range of areas, including philosophy, artificial intelligence, game theory, psychology and so on. What is knowledge? The answer to this question is often transformed into another question: What are the conditionsfor knowledge? For this, the most classic should be directly traced back to Plato. He believed knowledge is confirmed true belief, which also means:Agent knows $P$, and only if (I) $P$ is true, (II) Agent believes P, (III) Agent has good reason to believe P[2].

However, this traditional method waschallenged by Gettier. He put forward the counter example which meets the terms of the above definitionbut in fact agent does not know $P[3]$. Although it has been challenged, a new definition of knowledge has not yet emerged.And the'three conditions of knowledge'is still accepted by most people.

Correspondingly, in artificial intelligence, if the machine in every state explicitly encodes the fact into a sentence which can be interpreted as a formal language or can be derived from other formal sentences by the rules in logical system, it is seem as knowing the fact [1].In flied of science and technology, the study about knowledge reasoning is booming. Hintikka first time took the traditional modal logic technology into epistemic logic [4]. And Kripke model give a chance for semantic analysis to epistemic logic[5]. Since then, the study of knowledge has become an important research field in computer science, artificial intelligence, and game theory. Particularly, the computer scientists use the theory of epistemic logic to analyze multi-agent reasoning about knowledge and belief [6]. In artificial intelligence, researchers have tried to find out a tutoring systems abut computational and cognitive model concerning the nature of knowledge and communication [7]. Some are using the knowledge to model the intelligent agent's learning environment[8].And the research is from original static analysis to dynamic epistemic which refers to multi-agent knowledge, belief, action and other elements in interaction [9].Especially, dynamic epistemic logichas been used tomodel thehigh order information, the semantic update, and the 
machine default reasoning [10,11,12]. Thus,artificial intelligence is the main research field and the most important application of dynamicepistemic logic.

In order to further study the model of reasoning in communication, we can try different reasoning systems. For this, this paper, based on the knowledge reasoning in artificial intelligence,proposes a KSS system aboutbi-agent knowledge sharing which can explain how to change from the agent'sprivate knowledge tocommon knowledge. The KSS system is depicted in Fig. 1. This system can be used to simulate human-computer interaction, double robots, or the others.

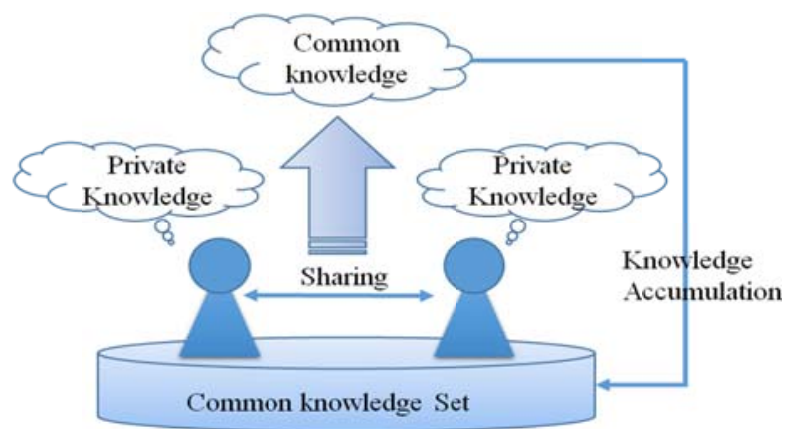

Figure 1. Knowledge Sharing and Interaction

\section{Single-agent Knowledge}

In fact, the single-agent knowledge is a complex subject.It is difficult to classify the knowledge from a single point of view. It can be the private knowledge, general knowledge, and backgroundknowledge, common knowledge, spatial knowledge, distributed knowledge, and so on.Surprisingly, in the field of artificial intelligence, thereis more popular in the research of distributed knowledge, especially the distributed reasoning [6]. The so-called distributed knowledge is a knowledge which is scattered in different agents but cannot becognized by agent itself. It is often through the sharing in the interaction, and become common knowledge [13]. Sometimes, beyond the agent, people can find an intelligent body (for example, God) thatis able to gain different distributed knowledge in different agents. But this intelligence is out of the agent itself. The significance of study lies in the ability of the machine to obtain the reason ability. While, in this paper, distributed knowledge is not the major knowledge in bi-agent communication, unless it can be characterized as private knowledge or common knowledge whichcan be cognized by agent itself.

But even so, many kinds of knowledgeare mostly can be classified intospecial knowledge and general knowledge. Special knowledge is only in someagents,but general knowledge is in each member [14]. General knowledge is just the first order of common knowledge;therefore it can be replayed by common knowledge [15].

\section{Bi-agent Common Knowledge}

As mentioned above, there are many kinds of knowledge involved, but from the agent's interaction, the reasoning generally only from the private knowledge, and the common knowledge for bi-agent interaction. Other knowledge can be regard as these two kinds of knowledge.

TheCommon Knowledge. David K. Lewis first time used common knowledge in his paper in 1969: It is a kind of knowledge that everyone knows, and everyone knows that everyone knows it, and so on [16]. Although Lewis first referred to the concept of common knowledge, its analysis was biased, non - formal, and somewhat confusing.In addition, economist Aumann in 1976 for the first time toformalize the definition of common knowledge which can eliminate infinite recursion, and the definition is widely applied in game theory, so that later people will be accustomed to think Aumann is the primary contributor to the study of common knowledge [17].If $E_{\mathrm{G}}, K_{i}$ and $C_{\mathrm{G}}$ respectively represent' the general knowledge of the group', 'single-agent knowledge' and 'group of common knowledge', then their relation as follows: 


$$
\begin{aligned}
& E_{G}=K_{1} \varphi \wedge \cdots \wedge K_{i} \varphi \wedge \cdots \wedge K_{N} \varphi . \\
& C_{\mathrm{G}}=\bigwedge_{k=1}^{\infty} E^{\kappa} \varphi
\end{aligned}
$$

Relativized Common Knowledge.In the process of research, it is found that if add the common knowledge operator to the public announcement logic, then a completeness proof with reduction axioms is impossible.Sincepublic announcement logic there is no reduction axiom for formulas [10]. If needs to use the reduction axiom to take plays in proof the system, we must revise the common knowledge, put forward the relativized common knowledge, makes it possible to use regulation axiomatic proof structure of the logic system [18].

If $C_{B}(\varphi, \psi)$ means 'relativized common knowledge', then the relation with the general common knowledge is as follows:

$$
C_{B} \varphi \equiv C_{B}(\varphi, \psi)
$$

In addition, the different from common knowledge to relativized common knowledge can be analyzed in detail as follow:

Definition 1 (Route):Let a finite set of group $B$, a set of possible world $W\left(w_{k} \in W, n>k \geq 0\right)$, a set of epistemic relation $R\left(R_{i} \in R, i \in B\right)$, the proposition $\varphi$, then the $\varphi$ route isasequence of possible worlds ' $w_{0}, w_{1}, \cdots, w_{k}, \cdots, w_{n}$ ', and $\varphi \in w_{k}$; the $B$ route is asequence of $\left(w_{k}, w_{k+1}\right) \in R_{i}$; the $B \cap \varphi$ route is a combinedsequence by $w_{k}$ and $R_{i}$ which means it is both $\varphi$ route and $B$ route.

Theorem 1(Basic theorem of canonical model ):Let $\left\langle W^{s}, R^{s}, V^{s}\right\rangle$ as acanonical model of a normal system, $S\left(\varphi, C_{B} \psi\right)$ is a formula for the system, $\Gamma$ is anany maximal consistent set $\left(\Gamma \in W^{s}\right)$, then:

$$
S\left(\varphi, C_{B} \psi\right) \in \Gamma \quad \text { iff } \quad(\mathrm{M}, \Gamma) \vDash S\left(\varphi, C_{B} \psi\right) .
$$

If we extend the public announcement system by add the common knowledge into it, proof the completeness of new system in Canonical model would involvebasic theorem of canonical model.By 'definition 1', we havethe result that (4) is equivalent to:

$$
S\left(\varphi, C_{B} \psi\right) \in \Gamma \quad \text { iff every } B \cap \varphi \text { route is } S(\varphi, \psi) \text { route }
$$

For the 'every $B \cap \varphi$ route is $S(\varphi, \psi)$ route' is not the 'every $B \cap \varphi$ route is $\psi$ route', if did not select the model which $\varphi$ is true in, then there are different route.

As analysis above, the following we will use relativized common knowledge to construct the system of relative knowledge. And unless otherwise specified, in the following, the 'common knowledge'refers to'relativized common knowledge'.

\section{The reasoning system about knowledge sharing(KSS): From single-agentprivate knowledge to bi-agent common knowledge}

According to the above analysis, many kinds of knowledge can be classified into private knowledge and common knowledge in the process of the bi-agent interaction. Therefore, KSS is a knowledge sharing system, which can explain how the private knowledge changes to common knowledge by sharing in the process of the interaction between bi-agent.

Language. Definition 2 (Language of KSS):Let a finite set of propositional variablesP, and a finite set of bi-agent $N=\{a, b\}$ be given. The language $\mathcal{L}_{K S S}$ is definedby the BNFas follows:

$$
\varphi::=p|\neg \varphi| \varphi_{1} \wedge \varphi_{2}\left|K_{i} \varphi\right| S\left(\varphi_{1}, \varphi_{2}\right) \mid C_{B}\left(\varphi_{1}, \varphi_{2}\right) .
$$

where, $\mathrm{p} \in \mathrm{P}, i \in \mathrm{N}$. ForKis the operator of 'know', and $K_{i} \varphi$ means 'agentiknow $\varphi$ ', or' $\varphi$ is agent $i$ 's knowledge'. Sis the operator of 'sharing', and $S\left(\varphi_{1}, \varphi_{2}\right)$ means ' $\varphi_{2}$ will hold after sharing $\varphi_{1}$ ',or 'if successfully sharing $\varphi_{1}$, then get $\varphi_{2}$ '.Cis the operator of 'relativized common knowledge', 
and $C\left(\varphi_{1}, \varphi_{2}\right)$ can read as ' $\varphi_{2}$ relativized to $\varphi_{1}$ is the common knowledge in double agent'.Moreover, $\check{\mathrm{K}}_{i} \varphi$ which is the dual of $K_{i} \varphi$ can read as'agenticonsiders it possible that $\varphi$ ', and $2\left(\varphi_{1}, \varphi_{2}\right)$ which is the dual of $S\left(\varphi_{1}, \varphi_{2}\right)$ means 'the sharing $\varphi_{1}$ can be made and after that $\varphi_{2}$ is true'.The otherabbreviations for the propositional connectives $\vee, \rightarrow$, and $\leftrightarrow$ are defined as usual.This language is interpreted in models for epistemic logic.

Semantics. Definition 3 (Epistemic model of KSS):A epistemic model of KSS is an ordered triple $M=\left\{W, R_{i}, V\right\rangle$, where $i \in N=\{a, b\}$, and

$W$ : a finite nonempty set of possible worlds.

$R_{i}: N \rightarrow \delta(W \times W)$ assigns an accessibility relation $R_{i}$ to each agent $i$.

$V:(W \times P) \rightarrow\{1,0\}$.

We wright $w \mathrm{R}_{i} w_{0}$ if $\left(w, w_{0}\right) \in \mathrm{R}_{i}$ for ' $w_{0}$ is accessible from $w$ for agent $i$ ', and $(\mathrm{M}, w)$ is an epistemic state or pointed model with $w \in \mathrm{W}$. Each $\mathrm{R}_{\mathrm{i}} \mathrm{is}$ an equivalence relation. As is usual for epistemic logic, which extends from multi-modal logic S5n. i.e., S5n is the subsystem of KSS, whose agentiknows true facts and is capable of both positive and negative introspection.

Definition 4 (Semantics of KSS):Let a finite set of propositional variables $P$, a finite set of bi-agent $N=\{a, b\}$, and a KSSepistemic model $(M, w)$ with $M=\left\{W, R_{i}, V\right\rangle b e$ given, and $w, v \in W, p \in P, i \in N=\{a, b\}$, and $\varphi, \psi \in \mathcal{L}_{K S S}$, then

$(\mathrm{M}, w) \vDash \mathrm{p} \quad$ iff $w \in \mathrm{V}(\mathrm{p})$

$(\mathrm{M}, w) \vDash \neg \varphi$ iff $(\mathrm{M}, w) \not \varphi$

$(\mathrm{M}, w) \vDash \varphi \wedge \psi$ iff $(\mathrm{M}, w) \vDash \varphi$ and $(\mathrm{M}, w) \vDash \psi$

$(\mathrm{M}, w) \vDash K_{i} \varphi$ iff $(\mathrm{M}, w) \vDash \varphi$ for all $v$ that $(w, v) \in \mathrm{R}_{i}$

$(\mathrm{M}, w) \vDash S(\varphi, \psi)$ iff $(\mathrm{M}, w) \vDash \varphi$ implies $\left(\mathrm{M}^{\varphi}, w\right) \vDash \psi$

$(\mathrm{M}, w) \vDash C_{B}(\varphi, \psi)$ iff $(\mathrm{M}, v) \vDash \psi$ for all $v$ that $(w, v) \in\left(\mathrm{R}_{B} \cap|\varphi|^{2}\right)^{*}$

Where $\mathrm{M}^{\varphi}$ can be seen as the relativized-model of $\mathrm{M}$, whoseelementsare the possible words of $\mathrm{M}$ where $\varphi$ is true, and $\mathrm{M}^{\varphi}:=\left\langle\mathrm{W}^{\varphi}, \mathrm{R}^{\varphi}, \mathrm{V}^{\varphi}\right\rangle$ is defined as follows:

$\mathrm{W}^{\varphi}:=\{v \in \mathrm{W} \mid \mathrm{M}, v \vDash \varphi\}$

$\mathrm{R}^{\varphi}:=\mathrm{R}_{i} \cap\left(\mathrm{W}^{\varphi} \times \mathrm{W}^{\varphi}\right)$

$$
\mathrm{V}^{\varphi}:=\mathrm{V} \cap \mathrm{W}^{\varphi}
$$

As noted above, for Semantic, the $\mathrm{M}^{\varphi}$ thatas a filter orrefining unit is an updated model in KSS.And the Semantic condition for the dual of $K_{i} \varphi$ and $S(\varphi, \psi)$ are as follows:

$(\mathrm{M}, w) \vDash \check{\mathrm{K}}_{i} \varphi$ iff for some $v$ that $(w, v) \in \mathrm{R}_{i}$ and $(\mathrm{M}, w) \vDash \varphi$

$(\mathrm{M}, w) \vDash 2(\varphi, \psi)$ iff $(\mathrm{M}, w) \vDash \varphi$ and $\left(\mathrm{M}^{\varphi}, w\right) \vDash \psi$

The otherabbreviations for the propositional connectives $\vee, \rightarrow$, and $\leftrightarrow$ are defined as usual. The $\left(\mathrm{R}_{B} \cap|\varphi|^{2}\right)^{*}$ is the reflexive transitive closure.

Theorem 2:Let a finite set of propositional variables $P$, and a epistemic model $(M, w)$ with $M=\langle W$, $\left.R_{i}, V\right\rangle$, be given, and $w, v \in W, p \in P, i \in N=\{a, b\}$, and $\varphi, \psi \in \mathcal{L}_{K S S}$, then

(a) $(\mathrm{M}, w) \vDash S(\varphi, p) \leftrightarrow(\varphi \rightarrow p)$

(b) $(\mathrm{M}, w) \vDash S(\varphi, \neg \psi) \leftrightarrow(\varphi \rightarrow \neg S(\varphi, \psi))$

(c) $(\mathrm{M}, w) \vDash S(\varphi, \psi \wedge \chi) \leftrightarrow(S(\varphi, \psi) \wedge S(\varphi, \chi))$

(d) $(\mathrm{M}, w) \vDash S\left(\varphi, K_{i} \psi\right) \leftrightarrow\left(\varphi \rightarrow K_{i} S(\varphi, \psi)\right)$

$(\mathrm{e})(\mathrm{M}, w) \vDash S(\varphi \wedge S(\varphi, \psi), \chi) \leftrightarrow S(\varphi, S(\psi, \chi))$

(f) $(\mathrm{M}, w) \vDash S\left(\varphi, C_{B}(\psi, \chi)\right) \leftrightarrow C_{B}(\varphi \wedge S(\varphi, \psi), S(\varphi, \chi))$

Proof:For any model $(\mathrm{M}, \mathrm{w})$

(a) $(\mathrm{M}, w) \vDash S(\varphi, p) \quad$ iff $(\mathrm{M}, w) \vDash \varphi \operatorname{implies}\left(\mathrm{M}^{\varphi}, w\right) \vDash p$

iff $(\mathrm{M}, w) \vDash \varphi \operatorname{implies}(\mathrm{M}, w) \vDash p$

iff $(\mathrm{M}, w) \vDash \varphi \rightarrow p$

(b) $(\mathrm{M}, w) \vDash S(\varphi, \neg \psi)$ iff if $(\mathrm{M}, w) \vDash \varphi$ then $\left(\mathrm{M}^{\varphi}, w\right) \vDash \neg \psi$

iff $\operatorname{if}(\mathrm{M}, w) \vDash \varphi$, then not $\left(\mathrm{M}^{\varphi}, w\right) \vDash \psi$

iff if $(\mathrm{M}, w) \vDash \varphi$, then $(\mathrm{M}, w) \vDash \varphi$ and not $\left(\mathrm{M}^{\varphi}, w\right) \vDash \psi$

iff $\operatorname{if}(\mathrm{M}, w) \vDash \varphi$, then not (if $(\mathrm{M}, w) \vDash \varphi$ then $\left(\mathrm{M}^{\varphi}, w\right) \vDash \psi$ )

iff $\operatorname{if}(\mathrm{M}, w) \vDash \varphi$, then not $(\mathrm{M}, w) \vDash S(\varphi, \psi)$ 


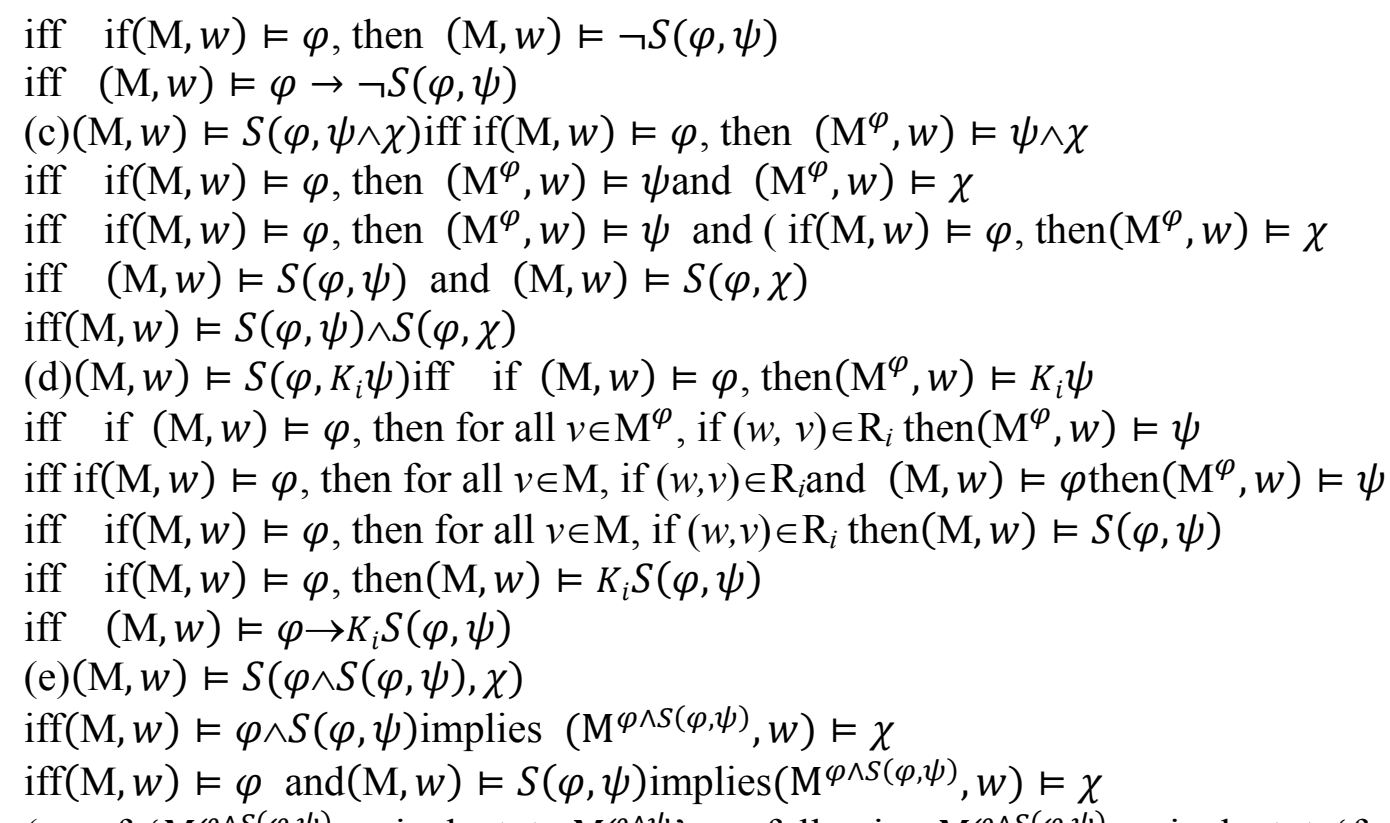

(proof ' $\mathrm{M}^{\varphi \wedge S(\varphi, \psi)}$ equivalent to $\mathrm{M}^{\varphi \wedge \psi}$ ' as following: $\mathrm{M}^{\varphi \wedge S(\varphi, \psi)}$ equivalent to' for all $v \in \mathrm{M}$, if ( $w$, $v) \in \mathrm{R}_{i}$ then $(\mathrm{M}, v) \vDash \varphi \wedge S(\varphi, \psi)$ ' iff 'for all $v \in \mathrm{M}$, if $(w, v) \in \mathrm{R}_{i}$ then $(\mathrm{M}, v) \vDash \varphi$ and $(\mathrm{M}, v) \vDash$ $S(\varphi, \psi)$ )' iff 'for all $v \in \mathrm{M}$, if $(w, v) \in \mathrm{R}_{i}$ then $(\mathrm{M}, v) \vDash \varphi$ and (if $(\mathrm{M}, v) \vDash \varphi$ then $\left.\left(\mathrm{M}^{\varphi}, v\right) \vDash \psi\right)$ ' iff 'for all $v \in \mathrm{M}$, if $(w, v) \in \mathrm{R}_{i}$ then $(\mathrm{M}, v) \vDash \varphi$ and $\left(\mathrm{M}^{\varphi}, v\right) \vDash \psi$ 'equivalent to $\mathrm{M}^{\varphi \wedge \psi}$.) $\operatorname{iff}(\mathrm{M}, w) \vDash \varphi$ and $(\mathrm{M}, w) \vDash S(\varphi, \psi) \operatorname{implies}\left(\mathrm{M}^{\varphi \wedge \psi}, w\right) \vDash \chi$

$\operatorname{iff}(\mathrm{M}, w) \vDash \varphi$ and $\operatorname{if}(\mathrm{M}, w) \vDash \varphi$ then $\left(\mathrm{M}^{\varphi}, w\right) \vDash \psi, \operatorname{implies}\left(\mathrm{M}^{\varphi \wedge \psi}, w\right) \vDash \chi$

$\operatorname{iff}(\mathrm{M}, w) \vDash \varphi$ and not $(\mathrm{M}, w) \vDash \varphi \operatorname{or}\left(\mathrm{M}^{\varphi}, w\right) \vDash \psi, \operatorname{implies}\left(\mathrm{M}^{\varphi \wedge \psi}, w\right) \vDash \chi$

$\operatorname{iff}(\mathrm{M}, w) \vDash \varphi$ and $\left(\mathrm{M}^{\varphi}, w\right) \vDash \psi$, implies $\left(\mathrm{M}^{\varphi \wedge \psi}, w\right) \vDash \chi$

iff $(\mathrm{M}, w) \vDash \varphi$ implies if $\left(\mathrm{M}^{\varphi}, w\right) \vDash \psi$ then $\left(\mathrm{M}^{\varphi \wedge \psi}, w\right) \vDash \chi$

iff $(\mathrm{M}, w) \vDash \varphi$ implies $\left(\mathrm{M}^{\varphi}, w\right) \vDash S(\psi, \chi)$

iff $(\mathrm{M}, w) \vDash S(\varphi, S(\psi, \chi))$

(f) $(\mathrm{M}, w) \vDash S\left(\varphi, C_{B}(\psi, \chi)\right)$ iff $(\mathrm{M}, w) \vDash \varphi$ implies $\left(\mathrm{M}^{\varphi}, w\right) \vDash C_{B}(\psi, \chi)$

iff $(\mathrm{M}, w) \vDash \varphi$ implies for all $v \in \mathrm{M}^{\varphi}$, if $(w, v) \in\left(\mathrm{R}_{B} \cap|\varphi|^{2}\right)^{*}$, then $\left(\mathrm{M}^{\varphi}, v\right) \vDash \chi$

iff $(\mathrm{M}, w) \vDash \varphi$ implies for all $v \in \mathrm{M}$, if $(\mathrm{M}, w) \vDash \varphi \operatorname{and}(w, v) \in\left(\mathrm{R}_{B} \cap|\varphi|^{2}\right)^{*}$, then $\left(\mathrm{M}^{\varphi}, v\right) \vDash \chi$

ifffor all $v \in \mathrm{M}$, if $(w, v) \in\left(\mathrm{R}_{B} \cap|\varphi \wedge S(\varphi, \psi)|^{2}\right)^{*},(\mathrm{M}, w) \vDash \varphi$ and $(\mathrm{M}, v) \vDash \varphi$, then $\left(\mathrm{M}^{\varphi}, v\right) \vDash \chi$

iff for all $v \in \mathrm{M}$, if $(w, v) \in\left(\mathrm{R}_{B} \cap|\varphi \wedge S(\varphi, \psi)|^{2}\right)^{*},(\mathrm{M}, v) \vDash \varphi$, then $\left(\mathrm{M}^{\varphi}, v\right) \vDash \chi$

iff for all $v \in \mathrm{M}$, if $(w, v) \in\left(\mathrm{R}_{B} \cap|\varphi \wedge S(\varphi, \psi)|^{2}\right)^{*}$, then, if $(\mathrm{M}, v) \vDash \varphi$, then $\left(\mathrm{M}^{\varphi}, v\right) \vDash \chi$

iff for all $v \in \mathrm{M}$, if $(w, v) \in\left(\mathrm{R}_{B} \cap|\varphi \wedge S(\varphi, \psi)|^{2}\right)^{*}$, then $(\mathrm{M}, v) \vDash S(\psi, \chi)$ $\operatorname{iff}(\mathrm{M}, w) \vDash C_{B}(\varphi \wedge S(\varphi, \psi), S(\varphi, \chi) \square$

For (d), it means that if $\varphi$ is true then before the sharing, the agent knows if he after successfully shared the knowledge, then $\psi$ holds. For (f), it claims that sharing knowledge is semantically equivalent to relativized common knowledge. For this, the relativized common knowledge after sharingcan be replaced by the relativized common knowledge before sharing.

Axiomatization. Definition 5 (Proof system for KSS):The proof system for KSS consists of all the following axioms:

(A1)All tautologies of propositional logic

(A2) $K_{i}(\varphi \rightarrow \psi) \rightarrow\left(K_{i} \varphi \rightarrow K_{i} \psi\right)$ knowledge distribution

(A3) $K_{i} \varphi \rightarrow \varphi \quad$ knowledge essence

(A4) $K_{i} \varphi \rightarrow K_{i} K_{i} \varphi \quad$ positive introspection

(A5) $\neg K_{i} \varphi \rightarrow K_{i} \neg K_{i} \varphi \quad$ negative introspection

(A6) $S(\varphi, p) \leftrightarrow(\varphi \rightarrow p)$ atoms

(A7) $S(\varphi, \neg \psi) \leftrightarrow(\varphi \rightarrow \neg S(\varphi, \psi)) \quad$ partial functionality

(A8) $S(\varphi, \psi \wedge \chi) \leftrightarrow(S(\varphi, \psi) \wedge S(\varphi, \chi))$ sharing distribution

(A9) $S\left(\varphi, K_{i} \varphi\right) \leftrightarrow\left(\varphi \rightarrow K_{i} S(\varphi, \psi)\right) \quad$ Ramsey axiom 
$(\mathrm{A} 10) S(\varphi \wedge S(\varphi, \psi), \chi) \leftrightarrow S(\varphi, S(\psi, \chi))$

$(\mathrm{A} 11) S\left(\varphi, C_{B}(\psi, \chi)\right) \leftrightarrow C_{B}(\varphi \wedge S(\varphi, \psi), S(\varphi, \chi))$

And the following rules:

(R1) $\varphi, \varphi \rightarrow \varphi \vdash \varphi$

$(\mathrm{R} 2) \varphi \vdash K_{i} \varphi$

(R3) $\psi \vdash S(\varphi, \psi)$

(R4) $\psi \vdash C_{B}(\varphi, \psi)$

Soundness and completeness. Theorem 3 (Soundness for KSS):For any formula $\varphi \in \mathcal{L}_{K S S}$, if $\vdash \varphi$ then $\vDash \varphi$.

Proof: For any formula $\varphi \in \mathcal{L}_{K S S}$, if $\vdash \varphi$, then according to the proof definition, it is by axioms and deduction rules of the systemand can obtain. So if $\vdash \varphi$, then it is only necessary to prove itsaxioms are valid, and its ruleskeep valid.It is easy to show there are valid for each axiom and deduction rules are keeping valid by $\mathrm{S} 5_{\mathrm{n}}$ and the theorem 2(a)-(f) above.

It is usually used to prove the completeness of the modal logic systems; in addition to that, there is another way, which is to use a translation rule, which can transform the dynamic logic of the updating operator to the static logic without updating the operator [19]. And in this process the concrete procedure is using reduction axioms to Hilbert-style proof system[18].

Before the formal proof of the completeness of the system, we need to give the relevant translation rules and related definitions as follows[20]:

Definition 6 (Translation of eliminating dynamic operator):The translation function ftakes a formula from the $\mathcal{L}_{K S S}$ to a formula in the $\mathcal{L}_{S 5 n}$ as follows:

$$
\mathrm{F}(u):=\left\{\begin{array}{cc}
f(\mathrm{~F}):=\mathrm{F}(u) & \text { if } \mathrm{F}=p \\
\neg f(\varphi) & \text { else if } \mathrm{F}=(\neg \varphi) \\
f(\varphi) \wedge f(\psi) & \text { else if } \mathrm{F}=(\varphi \wedge \psi) \\
K_{i} f(\varphi) & \text { else if } \mathrm{F}=\left(K_{i} \varphi\right) \\
C_{B}(f(\varphi), f(\psi)) & \text { else if } \mathrm{F}=\left(C_{B}(\varphi, \psi)\right) \\
f(\varphi \rightarrow \neg S(\varphi, \psi)) & \text { else if } \mathrm{F}=(S(\varphi, \neg \psi)) \\
f(\varphi \rightarrow p) & \text { else if } \mathrm{F}=(S(\varphi, p)) \\
f(S(\varphi, \psi) \wedge S(\varphi, \chi)) & \text { else if } \mathrm{F}=(S(\varphi, \psi \wedge \chi)) \\
f\left(\varphi \rightarrow K_{i} S(\varphi, \psi)\right) \text { else if } \mathrm{F}=\left(S\left(\varphi, K_{i} \psi\right)\right) & \text { else if } \mathrm{F}=\left(S\left(\varphi, C_{B}(\psi, \chi)\right)\right) \\
C_{B}(f(\varphi) \wedge f(S(\varphi, \psi)), f(S(\varphi, \chi))) & \text { else if } \mathrm{F}=(S(\varphi, S(\psi, \chi))) \\
f(S(\varphi \wedge S(\varphi, \psi), \chi)) &
\end{array}\right.
$$

Definition 7 (Modal complexity): Given any formula $F \in \mathcal{L}_{K S S}$, the Modal complexity of a formula is function Ctakes a formula from the $\mathcal{L}_{K S S}$ to Natural number set $N$, and noted as $\mathcal{C}(\mathrm{F})$ by the following rules:

$$
\mathcal{C}(\mathrm{F}):=\left\{\begin{array}{cc}
1 & \text { if } \mathrm{F}=\perp \\
1 & \text { else if } \mathrm{F}=p \\
1+\mathcal{C}(\varphi) & \text { else if } \mathrm{F}=(\neg \varphi) \\
1+\max (\mathcal{C}(\varphi), \mathcal{C}(\psi)) & \text { else if } \mathrm{F}=(\varphi \wedge \psi) \\
1+\mathcal{C}(\varphi) & \text { else if } \mathrm{F}=\left(K_{i} \varphi\right) \\
1+\max (\mathcal{C}(\varphi), \mathcal{C}(\psi)) & \text { else if } \mathrm{F}=\left(C_{B}(\varphi, \psi)\right. \\
(4+\mathcal{C}(\varphi)) \times \mathcal{C}(\psi)) & \text { else if } \mathrm{F}=(S(\varphi, \psi))
\end{array}\right.
$$

Theorem4 (Plaza's translation correctness):For any formula $\varphi \in \mathcal{L}_{K S S}$, there is 
Proof: Use the structure induction method.

(I) If $\varphi$ just a propositional variable like $p$, clearlyト $p \leftrightarrow p$.

(II) Inductive assumption: Above proposition is true when the modal complexity of formula $\varphi$ is less than or equal to n, i.e., $\vdash \varphi \leftrightarrow f(\varphi)$.

(III)If $\varphi$ is negation, conjunction or with individual epistemic operators, then by the inductive assumption, and the following sub-proof (i) the proposition holds, i.e. $\vdash \varphi \leftrightarrow f(\varphi)$.

Sub-proof (i): if $\psi \in \operatorname{sub}(\varphi)$, then $\mathcal{C}(\psi) \leq \mathcal{C}(\varphi)$.

Take structure induction method for $\varphi$. If $\varphi$ just a propositional variable like $p$, clearly $\mathcal{C}(\psi)=\mathcal{C}(\varphi)$. Inductive assumption: If $\psi \in \operatorname{sub}(\varphi)$, then $\mathcal{C}(\psi) \leq \mathcal{C}(\varphi)$. And $\chi \in \operatorname{sub}(\psi)$, then $\mathcal{C}(\chi) \leq \mathcal{C}(\varphi)$. If $\varphi$ is $\neg \psi$, then $\mathcal{C}(\neg \psi)=1+\mathcal{C}(\psi)$. If $\varphi$ is $K_{i} \psi$, then $\mathcal{C}\left(K_{i} \psi\right)=1+$ $\mathcal{C}(\psi)>\mathcal{C}\left(K_{i} \psi\right)$. If $\varphi$ is $\psi \wedge \chi$, then $\mathcal{C}(\psi \wedge \chi)=1+\max (\mathcal{C}(\psi), \mathcal{C}(\chi))>\mathcal{C}(\psi)$, and $\mathcal{C}(\psi \wedge \chi)=1+$ $\max (\mathcal{C}(\psi), \mathcal{C}(\chi))>\mathcal{C}(\chi)$. If $\varphi$ is $S(\psi, \chi)$, then $\mathcal{C}(S(\psi, \chi))=(4+\mathcal{C}(\psi)) \times \mathcal{C}(\chi)>\mathcal{C}(\psi)$, and $\mathcal{C}(S(\psi, \chi))=(4+\mathcal{C}(\psi)) \times \mathcal{C}(\chi)>\mathcal{C}(\chi)$.

(IV)If $\varphi$ is $S(\varphi, p)$, then by the inductive assumption, and the following sub-proof (ii) the proposition holds, i.e. $\vdash \varphi \leftrightarrow f(\varphi)$.

Sub-proof (ii): $\mathcal{C}(\varphi \rightarrow p) \leq \mathcal{C}(S(\varphi, p))$. Since by the definition above, $\mathcal{C}(\varphi \rightarrow p)=1+$ $\max (\mathcal{C}(\varphi), 2)<\mathcal{C}(S(\varphi, p))=(4+\mathcal{C}(\varphi)) \times \mathcal{C}(p)=4+\mathcal{C}(\varphi)$

(V)If $\varphi$ is $S(\varphi, \neg \psi)$, then by the inductive assumption, and the following sub-proof (iii) the proposition holds, i.e. $\vdash \varphi \leftrightarrow f(\varphi)$.

Sub-proof (iii): $\mathcal{C}(\varphi \rightarrow \neg S(\varphi, \psi)) \leq \mathcal{C}(S(\varphi, \neg \psi))$. Since by the definition

above, $\mathcal{C}(\varphi \rightarrow \neg S(\varphi, \psi))=1+\max (\mathcal{C}(\varphi), 2+(4 \mathcal{C}(\psi)+\mathcal{C}(\varphi) \mathcal{C}(\psi)))$

$<\mathcal{C}(S(\varphi, \neg \psi))=4+\mathcal{C}(\varphi)+4 \mathcal{C}(\psi)+\mathcal{C}(\varphi) \mathcal{C}(\psi)$

(VI)If $\varphi$ is $S(\varphi, \psi \wedge \chi)$, then by the inductive assumption, and the following sub-proof (iv) the proposition holds, i.e. $\vdash \varphi \leftrightarrow f(\varphi)$.

Sub-proof (iv): Since by the definition above, we have $\mathcal{C}(S(\varphi, \psi) \wedge S(\varphi, \chi)) \leq \mathcal{C}(S(\varphi, \psi \wedge \chi))$.

(VII) If $\varphi$ is $S\left(\varphi, K_{i} \psi\right)$, then by the inductive assumption, and the following sub-proof (v) the proposition holds, i.e. $\vdash \varphi \leftrightarrow f(\varphi)$.

sub-proof (v): Since by the definition above, we have $\mathcal{C}\left(\varphi \rightarrow K_{i} S(\varphi, \psi)\right) \leq \mathcal{C}\left(S\left(\varphi, K_{i} \psi\right)\right)$.

(VIII) If $\varphi$ is $S\left(\varphi, C_{B}(\psi, \chi)\right)$, then by the inductive assumption, and the following sub-proof (vi) the proposition holds, i.e. $\vdash \varphi \leftrightarrow f(\varphi)$.

Sub-proof (vi): Since by the definition above, we have $\mathcal{C}\left(C_{B}(\varphi \wedge S(\varphi, \psi), S(\varphi, \chi))\right) \leq$ $\mathcal{C}\left(S\left(\varphi, C_{B}(\psi, \chi)\right)\right)$.

(IX)If $\varphi$ is $S(\varphi, S(\psi, \chi))$, then by the inductive assumption, and the following sub-proof (vii) the proposition holds, i.e. $\vdash \varphi \leftrightarrow f(\varphi)$.

Sub-proof (vii): Since by the definition above,

$\mathcal{C}(S(\varphi \wedge S(\varphi, \psi), \chi))=(4+(1+\max (\mathcal{C}(\varphi),(4+\mathcal{C}(\varphi)) \times \mathcal{C}(\psi)))) \times \mathcal{C}(\chi)=(5+4 \mathcal{C}(\psi)+$ $\mathcal{C}(\varphi) \mathcal{C}(\psi)) \times \mathcal{C}(\chi)$

$$
\mathcal{C}(S(\varphi, S(\psi, \chi)))=(16+4 \mathcal{C}(\varphi)+4 \mathcal{C}(\psi)+\mathcal{C}(\varphi) \mathcal{C}(\psi)) \times \mathcal{C}(\chi)
$$

In the end, we have $\mathcal{C}(S(\varphi \wedge S(\varphi, \psi), \chi))<\mathcal{C}(S(\varphi, S(\psi, \chi)))$.

Theorem 5 (Completeness for KSS):For any formula $\varphi \in \mathcal{L}_{K S S}$, if $\vDash \varphi$ then $\vdash \varphi$.

Proof: (a) if $=\varphi$, then

(b) $\vdash \varphi \leftrightarrow f(\varphi)$ by the Lemma of Plaza's translation Correctness

$(\mathrm{c}) \vDash \varphi \leftrightarrow f(\varphi)$ by (b) and the Lemma of soundness theorem

$(d) \vDash f(\varphi) \quad$ by $(\mathrm{a}),(\mathrm{c})$, and Modus Ponens

$(\mathrm{e}) \vDash f(\varphi) \Longrightarrow \vdash f(\varphi)$ by the completeness of $\mathrm{S} 5_{\mathrm{n}}$, and $\mathrm{S} 5_{\mathrm{n}} \in \mathrm{KSS}$

(f) $\vdash f(\varphi) \quad$ by $(\mathrm{d}),(\mathrm{e})$, and Modus Ponens

The completeness of $\mathrm{S} 5 \mathrm{n}$ can refer to the classical modal logic books or periodical literature $[6,15,20,21]$.

Till then, main work that displays above has been completed. As we proof above, this system is sound and complete. It is can be used to make a detailed explanation on how agent to reason through the action of sharing in bi-agent interaction. For example, by axiom (A9), we can see that 
agent is sensibility because it understands it can know the new knowledge after the other agent's sharing action. And it is means if the sharing action holds, then the agent can know the new knowledge will be gain.

\section{Conclusions and further research}

In this paper, we analysis many kinds of knowledge, and classified them as the single-agentindividual knowledge, and bi-agent common knowledge. And then, we took private knowledge and relativized common knowledge as two kinds of operator for reasoning system which is about bi-agent knowledge sharing in interaction. And we also proof some useful related proposition properties. Through the KSS system, we obtain a logical methodto depict the process of bi-agent interaction from the privateknowledge to the common knowledge.According to KSS, we have found common knowledge is the key for keeping the interaction to go on. However, the action of sharing is a bridge of connecting private knowledge to common knowledge. But, how does private knowledge change? That is, how to form the internal iterate of private knowledge? After all, agent's knowledge base is different betweenbefore and after sharing. These questions will be our next work. The following research can be more complex, for instant, adds temporal operator in the current system, and then the proof for system would become more difficult, but also more useful and stronger reasoning ability.

\section{Acknowledgements}

This work was supported in part by the National Natural Science Foundation of China under Grant Nos.61273338, and in part by the Major Program of National Social Science Foundation of China under Grant 11\&ZD088.

\section{References}

[1] S.J. Rosenschein: New generation computing Vol.3(4)(1985), p.345-357

[2]Plato, in: Plato: The Collected Dialogues, edtied byE.Hamilton, H.Cairns.Princeton University Press,Princeton, New Jersey: (1961), p.853

[3]E.L. Gettier: analysis Vol.23(6)(1963), p.121-123

[4]J. Hintikka:Knowledge and belief: an introduction to the logic of the two notions(Cornell University Press,Ithaca 1962).

[5]S.A. Kripke: Mathematical Logic Quarterly Vol.9(5-6)(1963), p. 67-96

[6]R. Fagin, J.Y. Ronald, Y. Moses, M.Y. Vardi: Reasoning about knowledge(MIT press 1995).

[7]E. Wenger:Artificial intelligence and tutoring systems: computational and cognitive approaches to the communication of knowledge(Morgan Kaufmann, San Francisco, Califomia1987).

[8] V. Aleven: Artificial Intelligence Vol.150(1) (2003), p.183-237

[9] A. Baltag, S. Smets: Journal of Philosophical Logic (2015), p. 1-13

[10] J. Plaza:Synthese Vol.158(2) (2007), p.165-179

[11] F.Veltman: Journal of philosophical logic Vol.25(3) (1996), p.221-261

[12] J. Pearl: Probabilistic reasoning in intelligent systems: networks of plausible inference (Morgan Kaufmann, San Francisco, Califomia1988).

[13] J-J.C. Meyer, W. Van Der Hoek: Epistemic logic for AI and computer science(Cambridge University Press, Cambridge 2004). 
[14] P. Allo, J.P. Van Bendegem, and B. Van Kerkhove, in: The argument of mathematics, edtied by A. Aberdein, I.J. Dove, volume 30 of the series Logic, Epistemology, and the Unity of Science, chapter, 17, Springer Netherlands (2013), p.339-360

[15] J.Y.Halpern, Y. Moses: Artificial intelligence Vol.54(3) (1992), p.319-379

[16] D. Lewis: Conventions: A Philosophical Study(Harvard University Press, Cambridge, MA, 1969).

[17]R. J. Aumann: The annals of statisticsVol. 4(6) (1976), P. 1236-1239

[18]B. Kooi,J. van Benthem, in: AiML-2004: Advances in Modal Logic, Department of Computer Science, University of Manchester, Technical report series, UMCS-04-9-1(2004), p. 197-211.

[19] J. Van Benthem,J. Van Eijckand B. Kooi: Information and computation Vol. 204(11) (2006), p. $1620-1662$

[20] P. Blackburn, M. De Rijkeand Y. Venema: Modal Logic(Cambridge University Press,Cambridge 2001).

[21] H. vanDitmarsch, W. van der Hoekand B.Kooi:Dynamic epistemic logic(Springer Science \& Business Media 2008). 